Volume 30 (2021) 260-268

DOI: $10.24330 /$ ieja.969924

\title{
A NOTE ON SATURATED MULTIPLICATIVELY CLOSED SETS
}

\author{
Mehdi Badie \\ Received: 13 August 2020; Revised: 20 December 2020; Accepted: 20 December 2020 \\ Communicated by A. Çiğdem Özcan
}

\begin{abstract}
In this paper, we introduce and study $\mathcal{H}_{Y}$-s.m.c. and strong $\mathcal{H}_{Y^{-}}$ s.m.c. sets and give some connections between them and lattice ideals of $\mathcal{H}_{Y}$. Also, we introduce an ideal $R_{S}$, for each subset set $S$ of a ring $R$. We prove a ring $R$ is a Gelfand ring if and only if $R_{S}$ is an intersection of maximal ideals, for every s.m.c. set $S$ of $R$.
\end{abstract}

Mathematics Subject Classification (2020): 13A15, 54C40

Keywords: $\mathcal{H}_{Y}$-ideal, $\mathcal{H}_{Y}$-saturated multiplicatively closed set, closed ideal, $C(X)$, maximal ideal

\section{Introduction}

The concept of $\mathcal{H}_{Y}$-ideals as an extension of $z$-ideals and $z^{\circ}$-ideals is introduced and studied in [1] and [4]. In Section 2, we introduce (strong) $\mathcal{H}_{Y}$-s.m.c. sets, study lattice ideals of $\mathcal{H}_{Y}$, and give some connections between these concepts. We show that in each ring with the $h_{Y}$-property a subset $\mathcal{I}$ of $\mathcal{H}_{Y}$ is an ideal if and only if there is an $\mathcal{H}_{Y}$-s.m.c. set $S$ such that $\mathcal{I}=\mathcal{H}_{Y}(S)$. Also, we prove that every prime ideal of a ring $R$ is an $\mathcal{H}_{Y}$-ideal if and only if every s.m.c. subset of $R$ is an $\mathcal{H}_{Y}$-s.m.c. set. In Section 2 of paper [3], the concept of lattice ideal of $Z(X)$ (is called co-ideal) is studied, and the authors use this concept to give a new representation of closed ideals in the context of rings of real valued continuous functions. In Section 3, we generalize these concepts and improve some facts and proofs mentioned in that paper. The remainder of the current section is devoted to recalling some pertinent definitions.

Throughout this paper $R, \operatorname{Spec}(R), \operatorname{Max}(R)$ and $Y$ denotes a unitary commutative ring, the family of all prime ideals of $R$, the family of all maximal ideals of $R$ and a family of prime ideals, respectively. For any subset $\mathcal{P}$ of $Y$ and $S$ of $R$, we denote the kernel of $\mathcal{P}$ by $k(\mathcal{P})=\bigcap_{P \in \mathcal{P}} P$ and the hull of $S$ by $h_{Y}(S)=\{P \in Y$ : $S \subseteq P\}$. By $\mathcal{H}_{Y}$, we mean the family $\left\{h_{Y}(F): F\right.$ is a finite subset of $\left.R\right\}$. For each $S \subseteq R$ and $\mathcal{I} \subseteq \mathcal{H}_{Y}$, we denote $\left\{h_{Y}(F): F\right.$ is a nonempty finite subset of $\left.S\right\}$ and $\left\{a \in R: h_{Y}(a) \in \mathcal{I}\right\}$ by $\mathcal{H}_{Y}(S)$ and $\mathcal{H}_{Y}^{-1}(\mathcal{I})$, respectively. An ideal $I$ of a ring is 
called a (resp., strong) $\mathcal{H}_{Y}$-ideal if for each (resp., finite subset $F$ ) element $a$ of $I$ we have (resp., $\left.k h_{Y}(F) \subseteq I\right) k h_{Y}(a) \subseteq I$. A ring $R$ is said to have the $h_{Y}$-property if for every $a, b \in R$, there is some $c \in R$ such that $h_{Y}(a) \cap h_{Y}(b)=h_{Y}(c)$. Clearly, this is equivalent to say that for any finite subset $F$ of $R$, there is some $c \in R$ such that $h_{Y}(F)=h_{Y}(c)$. It is easy to see that if $R$ has the $h_{Y}$-property, then $\mathcal{H}_{Y} \mathcal{H}_{Y}^{-1}(\mathcal{I})=\mathcal{I}$, for each subset $\mathcal{I}$ of $\mathcal{H}_{Y}$.

Suppose that $S$ is a multiplicatively closed (for short "m.c.") set of $R$. By [6, Theorem 3.44], the family $\{I \subseteq R: I$ is an ideal of $R$ and $I \cap S=\emptyset\}$ has a maximal element and each maximal element of this family is prime. By $A^{c}$ we mean the set $R \backslash A$, for each subset $A$ of $R$. [6, Exercise 5.7] concludes that if $\left\{P_{\alpha}\right\}_{\alpha \in A}$ is the family of all maximal elements of $\{I \subseteq R: I$ is an ideal of $R$ and $I \cap S=\emptyset\}$, then $\bigcap_{\alpha \in A} P_{\alpha}^{c}$ is the smallest saturated multiplicative closed (for short "s.m.c.") set containing $S$. Thus if $S$ is an s.m.c. set, then $S=\bigcap_{\alpha \in A} P_{\alpha}^{c}$. It is clear that each maximal element of $\{I \subseteq R: I$ is an ideal of $R$ and $I \cap S=\emptyset\}$ is a minimal element of $\left\{I^{c} \subseteq R: I\right.$ is an ideal of $R$ and $\left.I \cap S=\emptyset\right\}$. We denote the family of all minimal elements of $\left\{I^{c} \subseteq R: I\right.$ is an ideal of $R$ and $\left.I \cap S=\emptyset\right\}$ by $\operatorname{Mim}(S)$. Hence $S$ is an s.m.c. set if and only if $S=\bigcap_{U \in \operatorname{Mim}(S)} U$.

Suppose that $L$ is a lattice, then an ideal $I$ of $L$ is a nonempty set of $L$ which satisfies

(a) $a \in L$ and $a \leqslant b \in I$ imply that $a \in I$.

(b) $a, b \in L$ implies that $a \vee b \in L$.

Evidently, a nonempty subset $I$ of $L$ is an ideal if and only if for every $a, b \in R$, we have $a \vee b \in I$ is equivalent to the fact that $a, b \in I$. In [3], an ideal of $Z(X)$ is called co-ideal.

The reader is referred to $[2,5,6,7,8]$ for undefined terms and notations.

\section{2. $\mathcal{H}_{Y}$-s.m.c. and strong $\mathcal{H}_{Y}$-s.m.c. sets}

In this section, we introduce and study $\mathcal{H}_{Y}$-s.m.c. and strong $\mathcal{H}_{Y}$-s.m.c. sets and give some relations between ideals of $\mathcal{H}_{Y}, \mathcal{H}_{Y}$-s.m.c. sets of $R$ and $\mathcal{H}_{Y}$-ideals of $R$.

Proposition 2.1. Let $S$ be an s.m.c. set of $R$. Then the following are equivalent.

(a) For any $a \notin S$ and $A \subseteq R, h_{Y}(a) \subseteq h_{Y}(A)$ implies that $A \cap S=\emptyset$.

(b) For any $a \notin S$ and $A \subseteq R, h_{Y}(a)=h_{Y}(A)$ implies that $A \cap S=\emptyset$.

(c) For any $a \notin S$ and $b \in R, h_{Y}(a)=h_{Y}(b)$ implies that $b \notin S$.

(d) For any $a \notin S$ and $b \in R, h_{Y}(a) \subseteq h_{Y}(b)$ implies that $b \notin S$.

(e) For any $a \notin S, k h_{Y}(a) \cap S=\emptyset$.

(f) For any $a \notin S$ and $A \subseteq R, k h_{Y}(a) \supseteq k h_{Y}(A)$ implies that $A \cap S=\emptyset$. 
(g) For any $a \notin S$ and $A \subseteq R, k h_{Y}(a)=k h_{Y}(A)$ implies that $A \cap S=\emptyset$.

(h) For any $a \notin S$ and $b \in R, k h_{Y}(a)=k h_{Y}(b)$ implies that $b \notin S$.

(i) For any $a \notin S$ and $b \in R, k h_{Y}(a) \supseteq k h_{Y}(b)$ implies that $b \notin S$.

(j) For any $a \in S$ and $b \in R, h_{Y}(a)=h_{Y}(b)$ implies that $b \in S$.

(k) For any $a \in S$ and $b \in R, h_{Y}(b) \subseteq h_{Y}(a)$ implies that $b \in S$.

(l) For any $a \in S$ and $b \in R, k h_{Y}(a)=k h_{Y}(b)$ implies that $b \in S$.

(m) For any $a \in S$ and $b \in R, k h_{Y}(b) \supseteq k h_{Y}(a)$ implies that $b \in S$.

Proof. (a) $\Rightarrow$ (b) $\Rightarrow$ (c) They are evident.

(c) $\Rightarrow$ (d) It is easy to see that $h_{Y}(a b)=h_{Y}(a) \cup h_{Y}(b)=h_{Y}(b)$. Since $S$ is an s.m.c. set of $R$ and $a \notin S$, it follows that $a b \notin S$. From this, we infer that $b \notin S$, by the assumption.

(d) $\Rightarrow$ (e) If $b \in k h_{Y}(a)$, then $h_{Y}(a) \subseteq h_{Y}(b)$. It follows that $b \notin S$, by the assumption. Consequently $k h_{Y}(a) \cap S=\emptyset$.

(e) $\Rightarrow$ (f) It is clear, by the fact that $A \subseteq k h_{Y}(A)$.

(f) $\Rightarrow$ (g) $\Rightarrow$ (h) They are evident.

(h) $\Rightarrow$ (i) It is clear that $k h_{Y}(a b)=k h_{Y}(a) \cap k h_{Y}(b)=k h_{Y}(b)$. Since $S$ is an s.m.c. set of $R$ and $a \notin S$, it follows that $a b \notin S$. From this, we conclude that $b \notin S$, by the assumption.

(i) $\Rightarrow$ (a) If $h_{Y}(a) \subseteq h_{Y}(A)$, then $h_{Y}(a) \subseteq h_{Y}(x)$, for every $x \in A$. Hence for every $x \in A$, we have $k h_{Y}(a) \supseteq k h_{Y}(x)$. This implies that $x \notin S$, by the assumption. From this, we infer that $A \cap S=\emptyset$.

(i) $\Leftrightarrow(\mathrm{c}),(\mathrm{j}) \Leftrightarrow(\mathrm{k}),(\mathrm{h}) \Leftrightarrow(\mathrm{l})$ and $(\mathrm{i}) \Leftrightarrow(\mathrm{m})$ They are clear.

Definition 2.2. An s.m.c. set $S$ of a ring $R$ is called an $\mathcal{H}_{Y}$-s.m.c. set if it satisfies one of the equivalent conditions of the above proposition.

Inspired by the concept of $z$-ideals (resp., $z^{\circ}$-ideals) we call an $\mathcal{H}_{Y}$-s.m.c. set a $z$-s.m.c. (resp., $z^{\circ}$-s.m.c. ) in case of $Y=\operatorname{Max}(R)$ (resp., $Y=\operatorname{Min}(R)$ ). Clearly, $S \subseteq \mathcal{H}_{Y}^{-1} \mathcal{H}_{Y}(S)$, for each $S \subseteq R$. Now by the above proposition, an s.m.c. set $S$ is an $\mathcal{H}_{Y}$-s.m.c. set if and only if $\mathcal{H}_{Y}^{-1} \mathcal{H}_{Y}(S)=S$.

Let $A$ and $B$ be two subsets of a ring $R$. One can see readily that $h_{Y}(A B)=$ $h_{Y}(A) \cup h_{Y}(B)$ and $B \subseteq k h_{Y}(A)$ if and only if $h_{Y}(B) \supseteq h_{Y}(A)$. These results lead to the following proposition.

Proposition 2.3. Let $S$ be an s.m.c. set of $R$. Then the following are equivalent.

(a) For every finite subset $F$ of $R$ disjoint from $S$ and $A \subseteq R, h_{Y}(F) \subseteq h_{Y}(A)$ implies that $A \cap S=\emptyset$. 
(b) For every finite subset $F$ of $R$ disjoint from $S$ and $A \subseteq R, h_{Y}(F)=h_{Y}(A)$ implies that $A \cap S=\emptyset$.

(c) For every finite subset $F$ of $R$ disjoint from $S$ and finite subset $G$ of $R$, $h_{Y}(F)=h_{Y}(G)$ implies that $G \cap S=\emptyset$.

(d) For every finite subset $F$ of $R$ disjoint from $S$ and finite subset $G$ of $R$, $h_{Y}(F) \subseteq h_{Y}(G)$ implies that $G \cap S=\emptyset$.

(e) For every finite subset $F$ of $R$ disjoint from $S, k h_{Y}(F) \cap S=\emptyset$.

(f) For every finite subset $F$ of $R$ disjoint from $S$ and $A \subseteq R, k h_{Y}(F) \supseteq$ $k h_{Y}(A)$ implies that $A \cap S=\emptyset$.

(g) For every finite subset $F$ of $R$ disjoint from $S$ and $A \subseteq R, k h_{Y}(F)=$ $k h_{Y}(A)$ implies that $A \cap S=\emptyset$.

(h) For every finite subset $F$ of $R$ disjoint from $S$ and finite subset $G$ of $R$, $k h_{Y}(F)=k h_{Y}(G)$ implies that $G \cap S=\emptyset$.

(i) For every finite subset $F$ of $R$ disjoint from $S$ and finite subset $G$ of $R$, $k h_{Y}(F) \supseteq k h_{Y}(G)$ implies that $G \cap S=\emptyset$.

Definition 2.4. An s.m.c. set $S$ of a $\operatorname{ring} R$ is called a strong $\mathcal{H}_{Y}$-s.m.c. set if it satisfies one of the equivalent conditions of the above proposition.

Inspired by the concept of strong $z$-ideals (resp., $z^{\circ}$-ideals), we call a strong $\mathcal{H}_{Y}$-s.m.c. set a strong $z$-s.m.c. (resp., $z^{\circ}$-s.m.c. ) in case of $Y=\operatorname{Max}(R)$ (resp., $Y=\operatorname{Min}(R))$. Clearly, every strong $\mathcal{H}_{Y}$-s.m.c. set is an $\mathcal{H}_{Y^{-}}$-s.m.c. set and if $R$ satisfies the $h_{Y}$-property, then the family of all $\mathcal{H}_{Y}$-s.m.c. sets and the family of all $\mathcal{H}_{Y}$-s.m.c. sets coincide. Evidently, for each prime ideal $P$ of $R, P$ is a (resp., strong) $\mathcal{H}_{Y}$-ideal if and only if $P^{c}$ is a (resp., strong) $\mathcal{H}_{Y}$-s.m.c. set. It is easy to show that the intersection of each family of (resp., strong) $\mathcal{H}_{Y}$-s.m.c. sets is a (resp., strong) $\mathcal{H}_{Y^{-}}$-s.m.c. set and thus the smallest $\mathcal{H}_{Y^{-}}$-s.m.c. set and strong $\mathcal{H}_{Y^{-}}$ s.m.c. set containing an s.m.c. set exist. Also, the union of a chain of (resp., strong) $\mathcal{H}_{Y}$-s.m.c. sets is a (resp., strong) $\mathcal{H}_{Y}$-s.m.c. set and, therefore any family of (resp., strong) $\mathcal{H}_{Y}$-s.m.c. sets contained in an $\mathcal{H}_{Y}$-s.m.c. set has a maximal element.

Lemma 2.5. Suppose that $S$ is a strong $\mathcal{H}_{Y}$-s.m.c. set of $R$ and $I$ is an ideal. If $I \cap S=\emptyset$, then $I_{S \mathcal{H}} \cap S=\emptyset$ and $I_{\mathcal{H}} \cap S=\emptyset$.

Proof. Suppose that $a \in I_{S \mathcal{H}}$, then by [1, Proposition 7.5], a finite subset $F$ of $I$ exists such that $k h_{Y}(a) \subseteq k h_{Y}(F)$. Thus $F \cap S=\emptyset$, since $I$ is a strong $\mathcal{H}_{Y}$-ideal, it follows that $k h_{Y}(F) \cap S=\emptyset$. So $k h_{Y}(a) \cap S=\emptyset$ and, therefore, $a \notin S$. Consequently $I_{S \mathcal{H}} \cap S=\emptyset$. By the fact that $I_{\mathcal{H}} \subseteq I_{S \mathcal{H}}$, it is clear that $I_{\mathcal{H}} \cap S=\emptyset$. 
Proposition 2.6. Suppose that $S$ is an s.m.c. set. $S$ is a strong $\mathcal{H}_{Y}$-s.m.c. set if and only if $U$ is a strong $\mathcal{H}_{Y}$-s.m.c. set, for each $U \in \operatorname{Mim}(R)$.

Proof. $(\Rightarrow)$ Suppose that $P^{c} \in \operatorname{Mim}(R)$. Since $P \cap S=\emptyset$, by Lemma 2.5, it follows that $P_{S \mathcal{H}} \cap S=\emptyset$, since $\left(P_{S \mathcal{H}}\right)^{c} \subseteq P^{c}$, by the minimality of $P^{c}$, it follows that $P_{S \mathcal{H}}=P$. Hence $P$ is a strong $\mathcal{H}_{Y^{-}}$-ideal and, therefore, $P^{c}$ is a strong $\mathcal{H}_{Y^{-}}$ ideal.

$(\Leftarrow)$ It is clear, by the fact that the intersection of any family of strong $\mathcal{H}_{Y}$-s.m.c. sets is a strong $\mathcal{H}_{Y}$-s.m.c. set.

Theorem 2.7. Suppose that $R$ has the $h_{Y}$-property. Then a subset $\mathcal{I}$ of $\mathcal{H}_{Y}$ is an ideal if and only if there is an $\mathcal{H}_{Y}$-s.m.c. set $S$ of $R$ such that $\mathcal{I}=\mathcal{H}_{Y}(S)$.

Proof. $(\Rightarrow)$ For each $a, b \in R$,

$$
\begin{aligned}
a b \in \mathcal{H}_{Y}^{-1}(\mathcal{I}) & \Leftrightarrow \quad h_{Y}(a b) \in \mathcal{I} \quad \Leftrightarrow \quad h_{Y}(a) \cup h_{Y}(b) \in \mathcal{I} \\
& \Leftrightarrow \quad h_{Y}(a), h_{Y}(b) \in \mathcal{I} \quad \Leftrightarrow \quad a, b \in \mathcal{H}_{Y}^{-1}(\mathcal{I})
\end{aligned}
$$

Thus $\mathcal{H}_{Y}^{-1}(\mathcal{I})$ is an s.m.c. set. Now suppose that $a \notin \mathcal{H}_{Y}^{-1}(\mathcal{I})$, then $h_{Y}(a) \notin \mathcal{I}$. If $h_{Y}(b) \supseteq h_{Y}(a)$, since $\mathcal{I}$ is an ideal of $\mathcal{H}_{Y}$, it follows that $h_{Y}(b) \notin \mathcal{I}$ and thus $b \notin \mathcal{H}_{Y}^{-1}(\mathcal{I})$. Therefore $S=\mathcal{H}_{Y}^{-1}(\mathcal{I})$ is an $\mathcal{H}_{Y}$-s.m.c. set of $R$. Since $R$ has the $h_{Y}$-property, it follows that $\mathcal{H}_{Y}(S)=\mathcal{H}_{Y} \mathcal{H}_{Y}^{-1}(\mathcal{I})=\mathcal{I}$.

$(\Leftarrow)$ Suppose that $S$ is an $\mathcal{H}_{Y}$-s.m.c. set, $\mathcal{H}_{1}, \mathcal{H}_{2} \in \mathcal{H}_{Y}(S)$ and $\mathcal{H}_{1} \in \mathcal{H}_{Y}$. Then two finite subsets $E$ and $F$ of $S$ exist such that $\mathcal{H}_{1}=h_{Y}(E)$ and $\mathcal{H}_{Y}=h_{Y}(F)$. Then $E F=\{x y: x \in E$ and $y \in F\}$ is a finite subset of $S$ and, therefore,

$$
\begin{aligned}
\mathcal{H}_{1} \cup \mathcal{H}_{2} & =h_{Y}(E) \cup h_{Y}(F) \\
& =\left[\bigcap_{x \in E} h_{Y}(x)\right] \cup\left[\bigcap_{y \in F} h_{Y}(y)\right] \\
& =\bigcap_{\substack{x \in E \\
y \in F}}\left[h_{Y}(x) \cup h_{Y}(y)\right] \\
& =\bigcap_{\substack{x \in E \\
y \in F}} h_{Y}(x y)=h_{Y}(E F) \in \mathcal{H}_{Y}(S)
\end{aligned}
$$

Now suppose that $\mathcal{H}_{1} \subseteq \mathcal{H}_{2} \in \mathcal{H}_{Y}(S)$. Since $R$ has the $h_{Y}$-property, there are $a, b \in R$ such that $h_{Y}(a)=\mathcal{H}_{1}$ and $h_{Y}(b)=\mathcal{H}_{2}$. Since $S$ is an s.m.c. set, it follows that $b \in \mathcal{H}_{Y}^{-1} \mathcal{H}_{Y}(S)=S$. Hence $h_{Y}(a b)=h_{Y}(a) \cup h_{Y}(b)=h_{Y}(b)$, since $S$ is an $\mathcal{H}_{Y^{-}}$ s.m.c. set, it follows that $a b \in S$, therefore $a \in S$ and thus $\mathcal{H}_{1}=h_{Y}(a) \in \mathcal{H}_{Y}(S)$. Consequently $\mathcal{H}_{Y}(S)$ is an ideal of $\mathcal{H}_{Y}$. 
Proposition 2.8. The following are equivalent.

(a) Every semiprime ideal of $R$ is a (resp., strong) $\mathcal{H}_{Y}$-ideal of $R$.

(b) Every prime ideal of $R$ is a (resp., strong) $\mathcal{H}_{Y}$-ideal of $R$.

(c) Every s.m.c. set of $R$ is a (resp., strong) $\mathcal{H}_{Y}$-s.m.c. set of $R$.

Proof. (a) $\Rightarrow$ (b) It is evident.

(b) $\Rightarrow$ (c) Suppose that $S$ is an s.m.c. set. By the assumption, there is a family $\left\{P_{\alpha}\right\}_{\alpha \in A}$ of prime $\mathcal{H}_{Y}$-ideals such that $S=\bigcap_{\alpha \in A} P_{\alpha}^{c}$. Now the fact that the intersection of any family of (resp. strong) $\mathcal{H}_{Y}$-s.m.c. sets is a (resp., strong) $\mathcal{H}_{Y^{-}}$ s.m.c. set implies that $S$ is a (resp., strong) $\mathcal{H}_{Y}$-s.m.c. set.

(c) $\Rightarrow$ (a) Suppose that $I$ is a semiprime ideal, then there is a family $\left\{P_{\alpha}\right\}_{\alpha \in A}$ of prime ideals such that $I=\bigcap_{\alpha \in A} P_{\alpha}$. By the assumption, $P_{\alpha}^{c}$ is a (resp., strong) $\mathcal{H}_{Y}$-s.m.c. set, for each $\alpha \in A$, and thus $P_{\alpha}$ is a (resp., strong) $\mathcal{H}_{Y}$-ideal, for each $\alpha \in A$. Since the intersection of any family of (resp., strong) $\mathcal{H}_{Y}$-ideals is a (resp., strong) $\mathcal{H}_{Y}$-ideal, it follows that $I$ is a (resp., strong) $\mathcal{H}_{Y}$-ideal.

Proposition 2.9. Suppose that $S$ is an s.m.c. set of $R$. If $Y$ is finite, then the following statements are equivalent.

(a) There is a subset $\mathcal{P}$ of $Y$ such that $S=\bigcap_{P \in \mathcal{P}} P^{c}$.

(b) $S$ is a strong $\mathcal{H}_{Y}$-s.m.c. set of $R$.

(c) $S$ is an $\mathcal{H}_{Y}$-s.m.c. set of $R$.

Proof. (a) $\Rightarrow$ (b) and (b) $\Rightarrow$ (c) They are evident.

(c) $\Rightarrow$ (a) Set $\mathcal{P}=\{P \in Y: S \cap P=\emptyset\}$. For each $P \in \mathcal{P}$, we have $S \subseteq P^{c}$. This yields $S \subseteq \bigcap_{P \in \mathcal{P}} P^{c}$. For each $P \in \mathcal{P}^{c}$, we have $S \cap P \neq \emptyset$. From this, we infer that there exists some $a_{P} \in S \cap P$ and thus

$$
\prod_{P \in \mathcal{P}^{c}} a_{P} \in S \cap k\left(\mathcal{P}^{c}\right) \neq \emptyset
$$

If $a \notin S$, then $k h_{Y}(a) \cap S=\emptyset$, so we have $k\left(\mathcal{P}^{c}\right) \nsubseteq k h_{Y}(a)$, by $(\star)$. This implies that $h_{Y}(a) \nsubseteq \mathcal{P}^{c}$ and, therefore, $h_{Y}(a) \cap \mathcal{P} \neq \emptyset$. It follows that $P \in \mathcal{P}$ exists such that $a \in P$, so $a \in \bigcup_{P \in \mathcal{P}} P$. It concludes that $a \notin \bigcap_{P \in \mathcal{P}} P^{c}$. From this, we conclude that $\bigcap_{P \in \mathcal{P}} P^{c} \subseteq S$ and consequently $S=\bigcap_{P \in \mathcal{P}} P^{c}$.

\section{An ideal corresponding to a subset}

If $S$ is a subset of $R$, then we denote $\{a \in R: 1-R a \subseteq S\}$, by $U_{S}$. Also, we denote the ideal generated by $U_{S}$, by $R_{S}$. In this section, we show that the intersection of each nonempty family of maximal ideals is of the form $R_{S}$, for some 
s.m.c. set $S$, and in Gelfand rings $R_{S}$ is an intersection of maximal ideals. Finally we improve some facts and simplify some proofs of [3].

Lemma 3.1. The following statements hold.

(a) $U_{S} \neq \emptyset$ if and only if $1 \in S$.

(b) If $S \subseteq T$ are two subsets of $R$, then $U_{S} \subseteq U_{T}$ and $R_{S} \subseteq R_{T}$.

(c) If $\left\{S_{\alpha}\right\}_{\alpha \in A}$ is a family of subsets of $R$, then $U_{\left(\bigcap_{\alpha \in A} S_{\alpha}\right)}=\bigcap_{\alpha \in A} U_{S_{\alpha}}$.

(d) If $M$ is a maximal ideal of $R$, then $U_{M^{c}}=M$ and $R_{M^{c}}=M$.

(e) Suppose that $S$ is a subset of $R$. If $S^{c}$ is contained in two distinct maximal ideals of $R$, then $R_{S}=R$.

Proof. (a), (b) and (c) They are clear.

(d) It is sufficient to show that $U_{M^{c}}=M$. For each $a \in R$,

$$
a \in M \quad \Leftrightarrow \quad(1-R a) \cap M=\emptyset \quad \Leftrightarrow \quad(1-R a) \subseteq M^{c} \quad \Leftrightarrow \quad a \in U_{M^{c}}
$$

Thus $M=U_{M^{c}}$.

(e) Suppose that $S^{c}$ is contained in two distinct maximal ideals $M_{1}$ and $M_{2}$, then $S$ contains $M_{1}^{c}$ and $M_{2}^{c}$. Hence part (b) implies that $R_{S}$ contains $R_{M_{1}^{c}}$ and $R_{M_{2}^{c}}$. Thus $R_{S}$ contains $M_{1}$ and $M_{2}$, by part (d). Consequently, $R_{S}=R$.

Theorem 3.2. If I is an ideal of a ring $R$, then $U_{I^{c}}=\bigcup_{M \in h_{M}(I)} M$.

Proof. For each $M \in h_{M}(I)$, we have $M^{c} \subseteq I^{c}$; so $U_{M^{c}} \subseteq U_{I^{c}}$, by Lemma 3.1(b). From this, Lemma 3.1(d) concludes that $\bigcup_{M \in h_{M}(I)} M \subseteq U_{I^{c}}$. Now suppose that $a \notin \bigcup_{M \in h_{M}(I)} M$. Then we have $I+a R=R$ and thus $(1-R a) \cap I \neq \emptyset$. This implies that $(1-R a) \not I^{c}$ and consequently $a \notin U_{I^{c}}$. It concludes that $U_{I^{c}} \subseteq \bigcup_{M \in h_{M}(I)} M$ and, therefore, $U_{I^{c}}=\bigcup_{M \in h_{M}(I)} M$.

Suppose that $\mathcal{M}$ is a family of maximal ideals, then $S=\bigcap_{M \in \mathcal{M}} M^{c}$ is an s.m.c. set. Hence $R_{S}=\bigcap_{M \in \mathcal{M}} M$, by Lemma 3.1 and Theorem 3.2. In the following corollary with the help of Lemma 3.1 and Theorem 3.2, we show that in Gelfand rings, $R_{S}$ is an intersection of maximal ideals. We recall that a ring is called a Gelfand ring if each prime ideal of $R$ is contained in exactly one maximal ideal.

Corollary 3.3. A ring $R$ is a Gelfand if and only if $R_{S}$ is an intersection of maximal ideals, for each s.m.c. set $S$ of $R$. In this case

$$
R_{S}=\bigcap\left\{M \in \operatorname{Max}(R): M \supseteq U^{c} \text {, for some } U \in \operatorname{Mim}(S)\right\} .
$$


We recall that in [3] each lattice ideal of $Z(X)$ is called co-ideal and for each lattice ideal of $Z(X)$ the following set is introduced:

$$
C_{\mathcal{I}}(X)=\{f \in C(X): Z(1-f g) \in \mathcal{I}, \text { for all } g \in C(X)\}
$$

For each closed subset $A$ of $\beta X$, write $S=\bigcap_{p \in A}\left(M^{p}\right)^{c}$, then $S$ is a $z$-s.m.c. and Corollary 3.3 implies that $M^{A}=C_{S}(X)=C_{Z^{-1}(S)}(X)$. Using Theorem 2.7, we conclude that $Z^{-1}(S)$ is an ideal of $Z(X)$. Consequently almost all the facts in [3, Section 2] are evident. By Theorem 2.7, for each ideal $\mathcal{I}$ of $Z(X)$ there is a $z$-s.m.c. set $S$ of $C(X)$ such that $\mathcal{I}=Z^{-1}(S)$. Clearly, $C_{\mathcal{I}}(X)=C_{S}(X)$. Now Corollary 3.3 concludes that $C_{\mathcal{I}}(X)=M^{A}$, for a closed subset $A$ of $\beta X$. Hence the converse of $[3$, Theorem 2.4] is also true.

Acknowledgement. The author would like to thank the referee for the valuable suggestions and comments.

\section{References}

[1] A. R. Aliabad, M. Badie and S. Nazari, An extension of $z$-ideals and $z^{\circ}$-ideals, Hacet. J. Math. Stat., 49(1) (2020), 254-272.

[2] M. F. Atiyah and I. G. Macdonald, Introduction to Commutative Algebra, Addison-Wesley Publishing Co., Reading, Mass.-London-Don Mills, Ont., 1969.

[3] F. Azarpanah, M. Ghirati and A. Taherifar, Closed ideals in $C(X)$ with different representations, Houston J. Math., 44(1) (2018), 363-383.

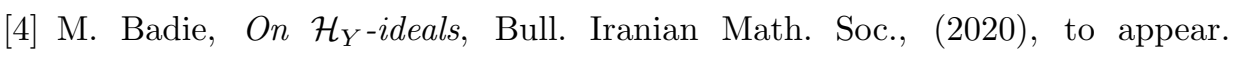
(https://doi.org/10.1007/s41980-020-00429-y)

[5] L. Gillman and M. Jerison, Rings of Continuous Functions, The University Series in Higher Mathematics D. Van Nostrand Co., Inc., Princeton, N.J.Toronto-London-New York, 1960.

[6] R. Y. Sharp, Steps in Commutative Algebra, Second edition, London Mathematical Society Student Texts, 51, Cambridge University Press, Cambridge, 2000.

[7] S. A. Steinberg, Lattice-Ordered Rings and Modules, Dordrecht, Springer, 2010.

[8] S. Willard, General Topology, Addison-Wesley Publishing Co., Reading, New York, 1970. 
Mehdi Badie

Department of Mathematics

Faculty of Science

Jundi-Shapur University of Technology

Dezful, Iran

e-mail: badie@jsu.ac.ir 